\title{
In vitro Studies on Sustained Release Suppository Formulations of Tiaprofenic Acid with Sucrose Fatty Acid Ester*
}

\author{
Sevgi Güngör*, Mine Orlu, Yıldız Özsoy, Ahmet Araman
}

Department of Pharmaceutical Technology, Faculty of Pharmacy, Istanbul University, Üniversite 34116, Istanbul, Turkey

\section{Abstract}

The objective of this study was to evaluate the performance of Sucro Ester 7 (sucrose distearate) as additive for preparing sustained release suppositories of tiaprofenic acid. Suppocire AIM (semi-synthetic glycerides) was used as suppository base and formulations were prepared containing different ratios of sugar ester: Suppocire AIM. Content uniformity, disintegration time and in vitro release characteristics of suppositories were investigated. Significant decrease in the extent of drug release was observed with the increase in the content of sugar ester, which was due to the longer disintegration time of suppositories.

\section{Keywords}

Tiaprofenic acid, sucrose fatty acid ester, sustained release suppository

\section{Introduction}

Suppository dosage forms are indicated for systemic action in paediatric patients and in patients who can not take or tolerate oral medication due to variety of reasons. The advantages of suppositories over other dosage forms are reduced side effects such as gastrointestinal irritation and avoidance of first-pass effect [1]. Sustained release suppositories have become the major interest of pharmaceutical scientists working on the design of long acting dosage forms. These forms are

\footnotetext{
*This study was presented at $11^{\text {th }}$ International Pharmaceutical Technology Symposium, September, 9-11, 2002, Istanbul-Turkey.
} 
preferred to conventional suppositories because they reduce the frequency of drug administration. Sustained release suppositories have been studied extensively using numerous additives and particular systems [2-4] and have been evaluated with in vitro release and bioavailability studies [5-6].

Sucrose fatty acid esters are biodegradable surfactants that can be manufactured in various hydrophilic-lipophilic properties using different fatty acids varying in their lipophilic chain length [7]. These surfactants were approved by FAOMHO in Japan, the USA, the EU, as food additives owing to their high safety and excellent properties [8]. Sucrose esters were also widely studied in pharmaceutical dosage forms as a penetration enhancer [9], emulsifying agent [10] and additive of sustained release suppositories [11].

Tiaprofenic acid (TA), a propionic acid derivative, is a nonsteroidal antiinflammatory drug used for the relief of pain and inflammation in rheumatic disorders such as osteoartiritis and rheumatoid arthritis [12]. TA has short half-life of 2 hours and it is given at least twice a day to maintain the therapeutic plasma level [13]. There is no marketed formulation of sustained release suppository formulation of TA.

The aim of our study was to assess the performance of Sucro Ester 7 (sucrose distearate, SE) as additive to formulate sustained release suppositories of TA to reduce daily dose and to minimize gastrointestinal disturbances.

Suppocire AIM (semi-synthetic glycerides, S-AIM) was used as suppository base and formulations were prepared containing different ratios of SE: S-AIM. Their content uniformity, disintegration time and in vitro release profiles were studied with this purpose. The release behaviour of TA from suppositories was evaluated by in vitro dissolution studies.

\section{Results and Discussion}

The standard deviation of content uniformity of suppositories prepared in this study was less than $3 \%$. These results showed that all the prepared suppositories 
satisfied the $\mathrm{Ph}$. Eur. $4^{\text {th }}$ Edition requirements [14]. As shown in Table 1, the disintegration tests indicated that the increase of SE content in the suppository formulations prolonged the disintegration time of the suppositories.

\begin{tabular}{|c|c|c|c|c|}
\hline $\begin{array}{c}\text { Formula } \\
\text { Code }\end{array}$ & $\begin{array}{c}\text { The ratio of } \\
\text { SE:S-AIM } \\
\text { (w/w) }\end{array}$ & $\begin{array}{c}\text { Tiaprofenic } \\
\text { acid (mg) }\end{array}$ & $\begin{array}{c}\text { Disintegration } \\
\text { time* (min.) }\end{array}$ & $\mathbf{r}^{* *}$ \\
\hline S1 & $0: 1$ & 30 & $5.0 \pm 0.1$ & 0.952 \\
\hline S2 & $1: 4$ & 30 & $16.7 \pm 5.2$ & 0.973 \\
\hline S3 & $1: 2$ & 30 & $48.0 \pm 13.0$ & 0.980 \\
\hline S4 & $2: 3$ & 30 & $65.0 \pm 19.7$ & 0.963 \\
\hline S5 & $1: 1$ & 30 & $210 \pm 14.1$ & 0.995 \\
\hline
\end{tabular}

"Data were expressed as the mean \pm SD $(n=6)$

"Correlation coefficient

Tab. 1. The content, disintegration time and correlation coefficient (Higuchi plot) of suppositories

The in vitro release profiles of TA from the suppositories as a function of time are shown in Figure 1. As can be seen from that figure, significant differences occurred among formulations with respect to percentage of the released drug.

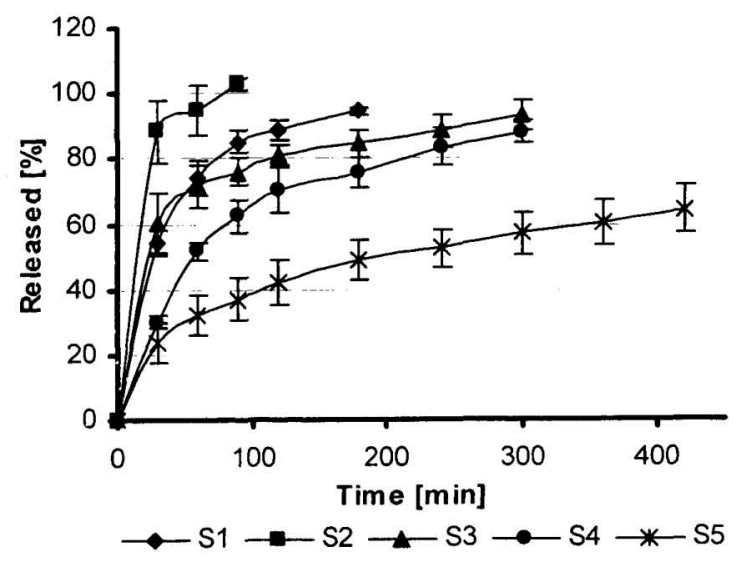

Fig. 1. In vitro release profiles of tiaprofenic acid from the suppositories 
A significant decrease $(p<0.001)$ in the extent of drug release was observed with the increase in the content of SE and this is attributable to the longer disintegration time of suppositories (Table 1). However, S2 having SE:S-AIM (1:4) ratio exhibited faster drug release than that of the $S 1$ suppository not containing SE. This could be explained by the fact that SE act as a surfactant [7] in lower ratios. About $60 \%$ of TA from S3 and S4 formulations was released in 1 hour and 1.5 hours, respectively. On the other hand, only $64.55 \pm 7.38 \%$ of TA was released in 7 hours from S5 which has SE:S-AIM (1:1) ratio. S5 significantly decreased the release extent of TA in comparison with $S 3(p<0.001)$ and $S 4(p<0.05)$.

The release mechanism of the drug from suppositories was examined by fitting the obtained dissolution data to Higuchi matrix model [15]. Figure 2 illustrates the cumulative amounts of drug released plotted against square-root of time. The plotted results indicate that suppository formulations showed linear relationship with a correlation of not less than 0.95 (Table 1). This observation implies that the drug released from all suppositories follows diffusion mechanism described by the Higuchi model, where the rate controling step is the process of diffusion through suppository base [15].

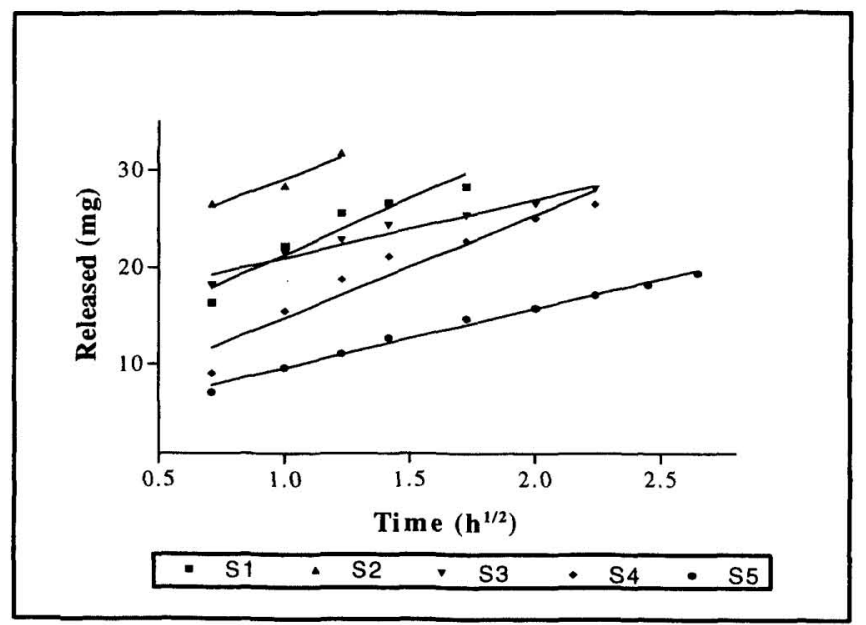

Fig. 2. In vitro release of tiaprofenic acid from suppositories as a function of the square root of time 
In conclusion from in vitro experiments, SE can be considered as a suitable additive for preparing sustained release suppository formulation of TA. To test the validity of these in vitro results and to determine the therapeutic level of the drug, thus allowing an appropriate formulation, the results should be supported by in vivo studies.

\section{Experimental}

\section{Materials}

Tiaprofenic acid was kindly supplied from Aventis Pharma, Turkey. Suppocire AIM (Semi-synthetic glycerides) and Sucro Ester 7 (sucrose distearate) were gifts from Gattefossé, France. All other chemicals used for analysis were of analytical grade.

\section{Preparation of suppositories}

Composition of suppository formulations (S1 - S5) containing different ratios of SE:(S-AIM) are given in Table 1. The content of TA in all studied suppositories was $30 \mathrm{mg}$. Briefly, S-AIM and SE were fused in a beaker in an oil-bath at $110 \pm 2^{\circ} \mathrm{C}$. TA was added and suspended in fused bases by using homogenisator (X 620 CAT, Germany). The mixture was cooled to $50 \pm 2^{\circ} \mathrm{C}$ and poured into suppository molds (1.0 $\mathrm{g}$ in weight), and the suppositories were kept at room temperature until completely cool. Then, they were refrigerated at $4 \pm 2^{\circ} \mathrm{C}$.

\section{Content uniformity}

The content uniformity was determined according to the procedures described in $\mathrm{Ph}$. Eur. $4^{\text {th }}$ Edition [14]. One suppository was accurately weighed it was suspended in $50 \mathrm{ml}$ of phosphate buffer solution (0.2 M, pH 7.4) and sonicated in ultrasonic bath at $40^{\circ} \mathrm{C}$ for 15 minutes. The volume of the suspension was adjusted to $100 \mathrm{ml}$ with $\mathrm{pH} 7.4$ phosphate buffer solution. Then the mixture was cooled to room temperature and filtered through $0.22 \mu \mathrm{m}$ Millipore ${ }^{\circledR}$ filter. $0.1 \mathrm{ml}$ of the filtrate was diluted to $10 \mathrm{ml}$ with the same buffer solution and samples were analysed 
spectrophotometrically at $316 \mathrm{~nm}$. Preliminary studies have shown that the presence of the suppository bases has no interference with the spectrophotometric method. TA contents were calculated using equation of standard curve which was plotted in the range of $0.5-10 \mathrm{mcg} / \mathrm{ml}\left(r^{2}=0.999\right)$. The experiment was repeated six times for each formulation.

\section{Disintegration time}

Disintegration time of suppositories was performed at $37 \pm 0.5^{\circ} \mathrm{C}$ in distilled water, with the apparatus described in Ph. Eur. $4^{\text {th }}$ Edition [14] from six parallel measurements.

\section{In vitro dissolution studies}

In vitro dissolution studies were carried out at $37 \pm 0.5^{\circ} \mathrm{C}$ in $900 \mathrm{ml}$ of phosphate buffer solution (0.2 M, pH 7.4) using special rotating basket (Vankel-122130, USA) at $50 \mathrm{rpm}$. At appropriate intervals, $1 \mathrm{ml}$ of samples was taken out with the medium replaced by fresh test solution. The content of TA in the samples was analysed with a spectrophotometer (Shimadzu UV-1601, Japan) at $316 \mathrm{~nm}$. Each experiment was repeated five times. Drug release mechanism from each suppository formulations was investigated according to Higuchi matrix model, which was plotted the amount of drug released versus square-root of time [15].

\section{Statistical analysis}

The data obtained from in vitro release studies was subjected to statistical analysis using a computer programme, PC-Instat, for a one-way analysis of variance following Student- Newman-Keuls multiple comparisons test. $P$ value of less than 0.05 was considered as evidence of a significant difference.

\section{Acknowledgement}

The authors are very grateful to Gattefossé, France for providing Suppocire AIM and sucrose fatty acid ester. The authors are also grateful to Aventis Pharma,Turkey for the generous supply of tiaprofenic acid. 


\section{References}

[1] Thoma K, editor.

Arzneiformen zur Rektalen und Vaginalen Applikation.

Frankfurt: Werbe und Vertriebsgesellschaft Deutscher Apotheker m.b.H., 1980.

[2] Kanke M, Tanabe E, Katayama $H$, Koda $Y$, Yoshitomi $H$.

Application of curdlan to controlled drug delivery. III. Drug release from sustained release suppositories in vitro.

Biol. Pharm. Bull. 1995;18:1154-8.

[3] Tarımcı N, Ermiş D.

Sustained release characteristics and pharmacokinetic parameters of ketoprofen suppositories using chitosan,

Int. J. Pharm. 1997;147:71-7.

[4] Schneeweis A, Muller-Goymann C C.

Controlled release of solid-reversed-micellar-solution (SRMS) suppositories containing metoclopramide- $\mathrm{HCl}$

Int. J. Pharm. 2000;196:193-6.

[5] Uzunkaya G, Bergişadi N.

In vitro drug liberation and kinetics of sustained release indomethacin suppository.

II Farmaco. 2003;58:509-12.

[6] Hosny E A, Al-Angary A A.

Bioavailability of sustained release indomethacin suppositories containing polycarbophil.

Int. J. Pharm. 1995;113:209-13.

[7] Garti N, Clement V, Leser M, Aserin A, Fanun M.

J. Molecular Liquids. 1999;80:253-96.

[8] Akoh C C.

Emulsification properties of polyesters and sucrose ester blends I: carbohydrate fatty acid polyesters.

J.Am. Oil Chem. Soc. 1992;69:9-19.

[9] Ganem-Quintanar A, Quintanar-Guerrero D, Falson-Rieg F, Buri P.

Ex vivo oral mucosal permeation of lidocaine hydrochloride with sucrose fatty acid esters as absorption enhancers.

Int. J. Pharm. 1998;173:203-10. 
[10]. Thevenin M.A., Grossiord J.L., Poelman M.C.

Sucrose esters/cosurfactant microemulsion systems for transdermal delivery: assessment of bicontinuous structures.

Int. J. Pharm. 1996;137:177-86.

[11] Nakajima T, Takashima $Y$, Furuya A, Ozawa $Y$, Kawashima $Y$. Indomethacin sustained-release suppositories containing sugar ester. Chem. Pharm. Bull. 1990;38:1027-31.

[12] Plosker G L, Wagstaff A J.

Tiaprofenic acid: A reappraisal of its pharmacological properties and use in the management of rheumatic diseases.

Drugs 1995;50:1050-75.

[13] Martindale, The Extra Pharmacopeia, $31^{\text {st }}$ Ed.

London: The Pharmaceutical Press, 1996:100.

[14] European Pharmacopoeia, $4^{\text {th }}$ Ed.

Strasbourg: Council of Europe, 67075, 2002:127-8.

[15] Higuchi T.

Mechanisms of sustained-action medication. Theoretical analysis of rate of release of solid drugs dispersed in solid matrices.

J. Pharm. Sci. 1963;52:1145-49. 